\title{
Effects of stimulus size, brightness and complexity upon EEG desynchronization
}

\section{GARRY BAKER AND ROBERT FRANKEN}

THE UNIVERSITY OF CALGARY

Measures of duration of EEG desynchronization were taken while $S s$ were exposed to a series of slides varying in complexity as well as size and brightness. There was a positive relation between duration of EEG desynchronization and level of complexity but no effect due to size or brightness. Significant habituation to temporal as well as spatial aspects of complexity were observed.

Various investigators have suggested that EEG desynchronization reflects increased cortical activity relating to vigilance, exploration, orientation, etc. (e.g., Roth, 1961; Berlyne \& Lewis, 1963; Voronin \& Sokolov, 1960). Berlyne \& McDonnell (1965) studied duration of EEG desynchronization using eight pairs of stimuli in which the second differed from the first in terms of (1) irregularity of arrangement, (2) amount of material, (3) heterogeneity of elements, (4) irregularity of shape, (5) incongruity, (6) number of independent units, (7) assymmetry, and (8) random redistribution. The second figure was defined as the more complex. Of the eight pairs, 1, 2, 5, and 8 differed significantly; the more complex by definition producing greater duration of EEG desynchronization. Since the duration was not significantly greater for half of the pairs used and since the pairs differed on a number of other attributes, the results might be a function of the unique nature of the stimuli used. In the present study, complexity is defined in terms of the number and magnitude of discontinuities in stimulation. Spatial complexity refers to discontinuities in stimulation produced by scanning the stimulus, while temporal complexity refers to the discontinuities in stimulation produced by the successive presentation of the stimuli. In the present study, spatial complexity was graduated along several steps using three basic figures (triangle, circle and square) and varying the number of overlapping figures per stimulus ( 1 to 10 inclusively). Temporal complexity was identified with the position of the slide in the series. A slide appearing early in the series is temporally more complex, compared with the adaptation stimuli, than a slide appearing later in the series. This is true irrespective of the amount of spatial complexity of the stimulus.

Several investigators have reported habituation of the EEG desynchronization to repetition of the same stimulus. Recovery has been reported when the stimulus is altered in some way such as size, brightness and temporal patterning (Berlyne, 1957; Wilson \& Wilson, 1959). Since duration of EEG desynchronization reflects habituation as well as recovery from habituation, both processes must be governed by the same stimulus variables. By controlling the novelty factor (addition of more, rather than new, material), manipulating position of slide in the series and varying amounts of complexity along a continuum of values, it is possible to determine whether Ss habituate to novelty, temporal complexity and/or spatial complexity. In order to control for the possible explanation that amount of photic stimulation governs desynchronization, size and brightness are also manipulated.

Subjects

Twenty-four University of Calgary students were randomly selected as Ss. All Ss with less than $25 \%$ or more than $90 \%$ Alpha recorded over the total recording time were discarded.

\section{Apparatus}

A Grass Model Seven polygraph was used to record EEG on one channel with a 7P3A preamplifier and a 7DAB driver amplifier. A second channel marked time in sec. (paper speed $25 \mathrm{~mm}$ per sec.) while a third registered the beginning and end of each stimulus pattern presentation. A monopolar recording was taken from the left occipital lobe of each $S$ by means of a single silver disc electrode. There was a reference electrode on the corresponding ear and a ground electrode attached to the arm.

The $S$ was placed in a reclining position facing a blank wall in a darkened room. The $E$ and apparatus were in an adjoining room from which the $S$ could be viewea through a one-way window. A $35 \mathrm{~mm}$ Kodak Carousal 800 projector was used to present the slides. Each slide was presented for 3 sec. with an interval of 17 sec.

Stimulus slides. The 60 stimulus slides used were variations of 10 basic figures of graduated complexity, number 1 being the simplest and number 10 being the most complex. The simplest was a triangle, the next most complex was a triangle with a circle superimposed on it. This procedure was continued until the most complex figure, number 10, was made up of four triangles, three circles and three squares randomly arranged on top of one another. Three figures rather than one are used in order to make the stimuli more discriminable. The slides were white figures on a black background and were of three sizes: small, medium, and large. The size ratio between large and medium, and medium and small was equal.

The brightness variable was manipulated by placing dark filter paper over a duplicate set of 30 slides 
Table 1. Mean Desynchronization Times

Level of

$\begin{array}{lllllllllll}\text { Complexity } & 1 & 2 & 3 & 4 & 5 & 6 & 7 & 8 & 9 & 10\end{array}$

Phase I $\quad 5.70 \quad 4.97 \quad 5.77 \quad 6.95 \quad 5.426 .31 \quad 8.098 .66 \quad 10.45 \quad 7.76$

Phase II $\quad 5.13 \quad 4.78 \quad 6.08 \quad 6.70 \quad 6.92 \quad 6.95 \quad 7.77 \quad 7.05 \quad 8.34 \quad 9.46$

involving the 10 complexities and three sizes. There were also a series of 10 slides, involving words on a dull background, that were used prior to the actual experimental slides to habituate the Ss to the sounds of the slide projector and presentation rate of the slides.

\section{Procedure}

After the Ss were positioned and the electrodes were in place, a short explanation of the experiment was given in order to relax the $S$. Ss were then told to close their eyes, the light was turned out and the E went into the next room to calibrate the polygraph. As soon as a satisfactory recording was obtained $S$ was told to open his eyes and informed that the experiment was about to begin. All Ss were asked to attempt to remain relaxed and to view the slides passively. Following the 10 habituation slides there was a $1 / 2$ min. delay before the 10 slides of Phase I. There was a 2 min. rest period between Phase I and II in order to facilitate recovery from habituation to the slides in Phase I. Within each phase of the experiment the order of presentation of the individual slides was randomized (Phase I: 9, 4, 8, 3, 7, 6, 2, 1, 10, 5; Phase II: $5,7,9,10,8,1,4,6,2,3)$. The order of presentation of the size of slide was reversed after one-half of each group had been run (e.g., the first three $\mathrm{Ss}$ in one group were shown the medium series first followed by the large series, but the last three Ss in that group were shown the large prior to medium).

Scoring. EEG scoring procedures were similar to that used by Berlyne \& McDonnell (1965) and Wilson \& Wilson (1959). A desynchronization was scored when the amplitude of the Alpha record dropped below $50 \%$ or the dominant frequency became greater than $13 \mathrm{cps}$. A return of three consecutive Alpha waves was considered a recovery. If no recovery was noted, a full score of $19 \mathrm{sec}$, was given. The full $20 \mathrm{sec}$. required for a complete sequence was not given because the noise of the apparatus changing the slides began $1 \mathrm{sec}$. before the slide actually appeared. The score recorded was the length of time in sec. from the disappearance to the return of the Alpha rhythm. Results

The data were treated statistically using an analysis of variance computer program (Lindquist III). The complexity variable was effective $(F=11.35$, df $=9 / 378$, $\mathrm{p}<.01$ ), while neither brightness or size had any significant effect on length of desynchronization. A test for critical difference between the 10 levels of complexity indicates no difference between adjacent slides $(d=1.65, p<.05)$ but a significant effect between the first five levels of complexity and the second five levels of complexity $(p<.05)$.

A correlated $t$ test was used to determine whether total desynchronization time differed between Phase I and Phase II. A significant decrease was noted between phases $(t=3.79, \mathrm{df}=1 / 23, \mathrm{p}<.01)$. This decrease indicates a significant habituation effect due to repetition.

\section{Discussion}

The results of the present study support Berlyne and McDonnell's complexity interpretation of EEG desynchronization but extend and modify this interpretation in certain respects. In addition to finding that more complex stimulus patterns evoke longer lasting desynchronization, our results indicate that Ss habituate to both temporal and spatial aspects of stimulus complexity. Desynchronization is longer to the more complex stimulus irrespective of the position of the stimulus in the series; however, desynchronization was longer if the stimulus appeared in the first phase as opposed to the second phase of the study. The reversals of the $3,5,6$, and 10 levels of complexity are probably a function of the differential position of the stimulus in the two series. Since novelty is controlled in the present study, the briefer durations cannot be attributed to the habituation of the response to novelty as suggested by Berlyne \& McDonnell (1965).

The failure to find effects due to size and brightness suggests that photic stimulation alone does not account for the results. Undoubtedly, if periodic changes in size and brightness were manipulated, the temporal change in size and brightness would have been effective. These findings support the interpretation that EEG desynchronization is governed by the same set of antecedents that appear to govern the vigilance, exploratory, and orientation responses of the organism.

\section{References}

Berlyne, D. E. Attention to change, conditioned inhibition (sI $)$ and stimulus satiation. Brit. J. Psychol., 1957, 48, 138-140. Berlyne, D. E., \& McDonnell, P. Effects of stimulus complexity on duration of EEG desynchronization. EEG clin. Neurophysiol., $1965,18,156-161$.

Berlyne, D. E., \& Lewis, J. L. Effects of heightened arousal on human exploratory behaviour. Canad. J. Psychol., 1963, 17, 398-410.

Roth, B. The clinical and theoretical importance of EEG rhythms corresponding to states of lowered vigilance. EEG clin. Neurophysiol., 1961, 13, 395-399.

Voronin, L. G., \& Sokolov, E. N. Cortical mechanisms of the orienting reflex and its relation to the conditioned reflex. In $\mathrm{H}$. H. Jasper and G. D. Sonirnov (Eds.), Moscow Colloquium on Electroencephalography of Higher Nervous Activity. EEG clin. Neurophysiol., 1960, (Suppl. 13), P. 355.

Wilson, $\mathbf{J}$. N., \& Wils $\mathrm{n}, \boldsymbol{w}$. P. The duration of human EEG arousal responses elicited by photic stimulation. EEG clin. Neurophysiol., 1959, 11, 85-91. 